\title{
Maximally Smooth Forward Rate Curves for Coupon Bearing Bonds
}

\author{
Hussain Abusaaq ${ }^{1}$, Paul M. Beaumont ${ }^{2 *}$ and Yaniv Jerassy-Etzion ${ }^{3}$ \\ ${ }^{1}$ Saudi Arabian Monetary Agency, Riyadh, Saudi Arabia \\ ${ }^{2}$ Department of Economics, Florida State Univeristy, Tallahassee, Florida 32306, United States \\ ${ }^{3}$ School of Economics and Business Administration, Ruppin Academic Center, Emek Hefer, Israel \\ Email: beaumont@f su.edu
}

\begin{abstract}
We present a fast and accurate algorithm to compute the maximally smooth instantaneous forward rate curve and the associated spot rate curve for the term structure of interest rates for coupon bearing bonds. The method produces zero pricing errors, constrains initial and terminal conditions of the spot and forward curves and produces the maximally smooth forward rate curve among the class of polynomial spline functions. The algorithm is simple enough to be quickly explained to traders and clients and flexible enough to be easily modified for different types of fixed income security markets. We illustrate the algorithm using on-the-run U.S. Treasury bonds for periods where the yield curve has a normal shape and also when it is inverted.
\end{abstract}

Keywords: Term structure of interest rates, yield curve, coupon stripping, curve interpolation, maximally smooth curves

\section{Introduction}

We present a new solution to an old problem - computing smooth spot, forward and discount rate curves from the term structure of on-the-run U.S. Treasury bills and bonds. We are able to reproduce observed security prices to arbitrary precision and we use the precise day count conventions used in the U.S. Treasury market. The algorithm is easily modified to capture the conventions used in other markets such as SWAP, corporate bond, or other sovereign bond markets.

Our primary motivation is the practical consideration that the algorithm should be fast, stable and easily understood by traders and clients while also being consistent with modern term structure theory. The algorithm uses a piecewise quartic polynomial interpolation of the forward curve and is an extension of [1], as corrected by [2]. Their method works only for zero coupon bonds so we extend the algorithm to include coupon bonds by simultaneously stripping the coupons and interpolating the spot curve. The interpolation step alone can be accomplished with a linear algorithm but the inclusion of simultaneous stripping leads to a highly nonlinear problem and it is critical that these steps be done simultaneously $[3,4]$. Our approach relies on an iterated linear algorithm that is simple to implement and very fast to compute while maintaining zero pricing errors and maximum smoothness of the interpolated curves. The maximum smoothness criterion ensures accurate prices for derivatives such as SWAPs [5] and that no artificial arbitrages will be introduced when pricing out-of-sample securities. We also correct some minor problems in [2] related to the terminal conditions of the spot and forward rate curves.

\section{Context and Related Literature}

There are five main goals that an ideal term structure model should achieve. The model should: (1) be theoretically sound; (2) produce no artificial arbitrages that are not present in the actual market; (3) be computationally tractable and fast; (4) produce smooth forward curves to accurately price derivative securities based upon the underlying bonds; (5) price the underlying bonds exactly.

This literature is vast so our intention is not to provide a thorough review but only to summarize the track of the literature that motivates our algorithm. Roughly, we may divide the literature into theoretical articles primarily directed at academic financial economists and applied articles primarily 
directed at financial practitioners. It is difficult to achieve all five goals so, as in most situations, there is a theory/practice trade-off in term structure modeling. What a modeler is willing to compromise on depends upon their primary focus. As a rough rule of thumb, theorists' order of importance is from the top down and practitioners' order of importance is from the bottom up on the list of goals.

Early theory papers offered hypotheses to explain the shape of the yield curve [6] but were easily rejected empirically [7]. A major theoretical breakthrough came with the development of dynamic stochastic general equilibrium models in financial economics [8]. This provided a framework for term structure models that were consistent with an underlying asset pricing kernel derived from investor preferences [9,10]. These models did a good job of achieving goals 1 (theoretically sound), 2 (no-arbs) and 4 (smooth forwards) but failed rather spectacularly at goals 3 (tractability) and 5 (accurate pricing).

Pricing errors motivated a series of papers with flexible functional forms that attempted to capture the various shapes commonly observed in the yield curve $[11,12,13,14,15,16,17,18]$. These models were more or less consistent with financial economic theory and did quite well with goal 3 (tractability) and reasonably well at goal 4 (smooth forwards) but still proved unable to price fixed income securities with sufficient accuracy for practitioners' purposes and they also could not guarantee the absence of false arbitrage signals in the term structure.

The problem of accurate pricing was essentially due to the fact that models with only a few factors, such as level, slope and curvature, have too few free parameters to exactly price all of the underlying securities. This problem was elegantly solved, in theory, by [19] who modeled the forward curve directly with an infinite order stochastic differential equation. Their model came very close to satisfying all five of our goals but proved to be very difficult to implement in practice [20] in a tractable way.

Another term structure modeling breakthrough was the development of affine models which could guarantee no arbitrages by imposing additional restrictions in the optimization problem [21]. This innovation led to a series of papers that modified the various one, two and three factor models to ensure no arbitrages $[22,23,24,25,26]$. The fundamental problem of having too few free parameters to model too many securities to price remains with these models, however, so the practitioners' primary goal of accurate pricing is not satisfied. Additionally, the arbitrage-free conditions typically imposed in these affine models are highly non-linear in the model parameters so their solutions require quite complex and often very slow algorithms.

The persistent problem of accurate pricing led to, in a sense, a "return to the basics" in term structure modeling among practitioners. Several early papers, before the introduction of the stochastic general equilibrium theories in vogue today, relied upon relatively simple numerical methods such as cubic splines for the spot curve [27] and cubic exponential splines for the discount curve [28] to fit and price securities along the term structure. These models are able to price the underlying securities very accurately because there are at least as many parameters to fit as there are securities to price. Unfortunately, these spline methods produced forward curves which were not twice differential at the knots [29]. The problem is that if you use a cubic spline to model the spot curve then that curve will be twice differential at the knot points. However, the forward curve, which is derived from the derivative of the spot curve, will only be once differentiable at the knots. The resulting kinks in the first derivative of the forward curve may signal false arbitrages in certain types of derivatives, such as swaptions (SWAP options), which do not really exist in the actual markets. As noted by [29], smoother forward curves would improve pricing performance.

Consequently, several enhanced versions of spline methods were introduced [30,3,31,32,33,34,35] including using genetic algorithms [36,37] and dynamic factor analysis with factor smoothing [38]. Most of these algorithms had improved pricing accuracy but suffered from being complex to implement and slow to execute. One very pragmatic approach $[1,5]$ was to note that, if we desire the forward curve to be everywhere twice differentiable, then the spot curve must be at least everywhere four times differentiable. Several of the flexible functional forms noted above satisfied this condition but none had sufficient parameters to price accurately. By restricting the function space to the class of piece-wise polynomials with a knot for each security, [1] was able to prove that a fourth order polynomial spline function for the spot curve would produce the maximally smooth forward curve within this space of functions. As long as the number of knots is at least as large as the number of securities the algorithm will produce very accurate pricing. Furthermore, if we restrict our attention to securities with no intermediate payments such as zero-coupon bonds, this algorithm can be reduced to a linear set of equations so it is very fast 
to solve. The algorithm of [1] has been slightly modified [2] and extended [39,40], but the main critique of this approach, that it cannot be used for coupon bearing bonds, such as U.S. Treasury bonds with coupon payments, remained.

Many of the term structure models and algorithms discussed above have been compared in $[41,4]$. The general conclusion is that the small factor and affine models produce very smooth, perhaps too smooth, forward curves but are generally computationally expensive and fail to price accurately enough to meet the needs of practitioners. Algorithms based upon genetic algorithms and other large space search methods are slow and can often produce unreliable or erratic results. Polynomial spline methods can be very fast and accurate but special care must be taken to ensure no arbitrage and twice differentiable forward curves.

Our approach is to extend the quartic polynomial spline method [5] to handle coupon bearing bonds. To maintain speed and accuracy we will impose an exactly identified parameter space so that a sequence on linear models can be used to solve the non-linear pricing problem. The no arbitrage conditions can be imposed with additional constraints on the polynomial splines. What we must sacrifice is strict adherence to modern general equilibrium asset pricing theory. We can only assert that, because our derived discount function is everywhere at least twice differentiable and monotone decreasing, our model is consistent with some version of an equilibrium pricing kernel even though we cannot derive that kernel from first principles. Consequently, our approach is firmly in the camp of the practitioners.

\section{Background of the Problem and Preview of the Results}

The primary complication, and what makes modeling the term structure an interesting problem, is that we only observe prices and yields-to-maturity of a finite set of securities from several different markets including on-the-run and off-the-run treasuries, ${ }^{1}$ corporate bonds, LIBORs, SWAP rates, and various derivatives such as TIGR strips. Each of these markets has its own liquidity and risk characteristics that must be accounted for when constructing the discount function. We choose to model the on-the-run U.S. government treasuries because they are highly liquid and they have very low and uniform credit risk and are often used in conjunction with option adjusted spreads to price other fixed income securities $[33,42]$.

In the U.S. Treasury market we observe zero-coupon Treasury bills of maturities one, three, six and twelve months and semi-annual coupon paying Treasury bonds of maturities two, three, five, seven, ten and thirty years. As an example, Table 1 presents the yield curve data for the on-the-run U.S. Treasuries as of February 10, 2012. The first column of Table 1 gives the maturity date of the bill or bond; the second column gives the annualized percent coupon rate (paid semi-annually) which is zero for the pure discount bills $;^{2}$ the third column gives the quoted asking prices; ${ }^{3}$ and column four gives the yield-to-maturity in percents using the ask price and appropriate day count conventions.

Our goal is to compute from these ten observations the continuous discount function $\delta(T)$, spot function $y(T)$, and instantaneous forward function $f(T)$ for a specific settlement date such as $2 / 10 / 2012$ for all maturities $T \geq 0$ such that the curves are consistent with the observed market data in Table 1 .

The discount rate $\delta(T)$ is the present discounted value at the settlement date of a $\$ 1$ payment at time $T$. By definition, $\delta(0)=1$ and the discount rate declines monotonically to zero as $T \rightarrow \infty$. The spot rate $y(T)$ is the average rate (in decimals) at which the cash-flow at date $T$ must be discounted to achieve the discounted price $\delta(T)$. Note that the spot rate equals the reported yield-to-maturity for pure discount or zero-coupon bills ${ }^{4}$ but not for coupon bonds. It is conventional to report the spot rate in annualized percents.

\footnotetext{
${ }^{1}$ On-the-run treasuries are the most recently auctioned securities and off-the-run treasuries are securities from previous auctions.

${ }^{2}$ For example, the holder of a 30 year bond with $\$ 100$ face value would receive a coupon payment of $\$ 1.56$ every six months and the holder of the one year bill simply receives the face value at maturity.

3 The quoted price is the ask clean price which is the broker's selling price of the bond not including the accrued interest due to the current bond holder. Some sources quote the dirty price which includes the accrued interest since the previous coupon payment. The broker's purchase price is the somewhat lower bid price. The bid-ask spread constitutes the broker's profits.

${ }^{4}$ In fact, the reported yield-to-maturity for bills uses a discrete bond yield equivalent method but we will compute our spot rates using continuous compounding which will cause small deviations between the two values.
} 
Table 1. Observed yield curve data for on-the-run U.S. Treasuries on February 10, 2012. The first column is the maturity date (mm/dd/yyyy), column two is the percent coupon rate, column three is the quoted asking price, and column four is the percent ask yield-to-maturity.

\begin{tabular}{cccc}
\hline Maturity Date & Coupon & Ask Price & Ask Yield \\
\hline $03 / 08 / 2012$ & 0.00 & 0.03 & 0.03 \\
$05 / 10 / 2012$ & 0.00 & 0.07 & 0.08 \\
$08 / 09 / 2012$ & 0.00 & 0.11 & 0.11 \\
$02 / 07 / 2013$ & 0.00 & 0.14 & 0.14 \\
$01 / 31 / 2014$ & 0.25 & 99.98 & 0.26 \\
$02 / 15 / 2015$ & 0.25 & 99.66 & 0.36 \\
$01 / 31 / 2017$ & 0.88 & 100.37 & 0.80 \\
$01 / 31 / 2019$ & 1.25 & 99.33 & 1.35 \\
$02 / 15 / 2022$ & 2.00 & 100.34 & 1.96 \\
$02 / 15 / 2042$ & 3.12 & 100.11 & 3.12 \\
\hline
\end{tabular}

Define $F(n, T), n \leq T$, to be the current price of a forward contract to deliver a $T-n$ maturity pure discount bond at date $n$ that matures at date $T$. To avoid arbitrage, it must be the case that

$$
F(n, T)=\frac{\delta(T)}{\delta(n)} .
$$

The instantaneous forward rate $f(T)$ is the forward rate $F(n, T)$ as $n \rightarrow T$. Forward curves of any horizon $n$ can be constructed from the instantaneous forward curve so, hereafter, we will refer to the instantaneous forward curve simply as the forward curve. To avoid arbitrage, the forward curves must be everywhere non-negative but need not be monotonic.

Assuming continuous compounding, the relationships between the discount, spot and instantaneous forward curves are given by

$$
\begin{aligned}
& \delta(T)=\exp (-y(T) T)=\exp \left(-\int_{0}^{T} f(t) d t\right), \\
& f(T)=y(T)+T \frac{d}{d T} y(T)=\frac{d}{d T}(-\ln \delta(T)),
\end{aligned}
$$

and

$$
y(T)=\frac{1}{T} \int_{0}^{T} f(t) d t=-\frac{1}{T} \ln \delta(T) .
$$

The nature of these equations lead us to think of $-\ln \delta(T)$ as the "level" curve, the spot function $y(T)$ as the "average" curve, and the forward function $f(T)$ as the "marginal" curve. ${ }^{5}$

Note that, from $(2 \mathrm{~b}), f(0)=y(0)$ so that the spot and forward curves must begin at the same rate at zero maturity. It is also reasonable to expect that the spot and forward curves should be well-behaved as maturities increase beyond that of the longest observed security. This is particularly important if we need to price, say, a forward contract on a thirty-year bond five or ten years hence. Consequently, we assume that

$$
\lim _{T \rightarrow \infty} \frac{d}{d T} y(T)=\lim _{T \rightarrow \infty} \frac{d}{d T} f(T)=0
$$

\footnotetext{
${ }^{5}$ See [34] for a more complete discussion of the relationships between the discount, spot and forward curves.
} 
so that

$$
\lim _{T \rightarrow \infty} y(T)=\lim _{T \rightarrow \infty} f(T) .
$$

The point of our algorithm is to produce maximally smooth forward curves so the most direct way to preview and understand our results is to examine the output of the algorithm in terms of the fundamental spot and forward curves generated. Figure 1 displays the spot and instantaneous forward rate curves computed by our algorithm, described in Section 4, for the yield curve on February 10, 2012 whose data is given in Table 1. The spot curve is the solid black line, the instantaneous forward rate curve is the dotted line, and the observed ask yields are indicated by the small circles. The top panel in the figure shows all maturities from zero to 30 years which is the longest maturity bond in our data. The slope of the spot curve is zero at maturity 30 years so the forward curve will decay monotonically toward the spot curve for maturities greater than 30 years. The bottom figure zooms in on the short maturities between zero and two years of the curves. This is the region of greatest interest for short-term portfolio managers who typically do not hold portfolios for more than two years and so need an accurate estimate of the discount function $(2 \mathrm{a})$ within that date range.

It is worth remembering that there is no unique set of discount, spot and forward curves that are consistent with the observed security prices. The "true" curves are unobserved. As we show below, the forward curve $f(t)$ shown in Figure 1 is the smoothest curve possible among the class of polynomial spline functions that also satisfy the conditions of exact bond pricing, no arbitrage, and boundary conditions for zero and infinite maturity bonds. By contrast, the method of linear bootstrapping of the spot curve, perhaps the most commonly used technique to construct forward curves (see for instance, [42] or [43]), produces piecewise linear segments between the knots of the spot curve $y(t)$. Although this spot curve is continuous, it is non-differential at the knots so that, by $(2 \mathrm{~b})$, the method would produce forward curves with discontinuities at the knot points. Since most derivative assets are priced off of the forward curve, these discontinuities would imply arbitrage opportunities for, say, SWAP products structured on either side of the knot point. Although linear bootstrapping is the extreme case, any stripping/interpolation method that produces too much curvature in the forward rates will signal arbitrage opportunities that likely do not exist in actual markets.

Finally, Figure 1 also illustrates several important properties of the curves. First, the forward curve $f(t)$ is generally more volatile than the spot curve $y(t)$. This motivates the approach of directly modeling the forward curve rather than the spot or discount curves since constructing a smooth forward curve assures smooth spot and discount curves. Second, the term structure at this date produces an increasing spot curve and the forward curve lies everywhere above the spot curve. If the spot curve has local maxima and minima the forward curve would intersect the spot curve at those points. Finally, our forward curve has a slope of zero at maturity $T=30$ years, the longest maturity bond. If we were to extend the curves beyond $T=30$ we would observe that the forward curve remains flat and that the spot curve will asymptotically approach the forward curve. This asymptotic behavior is non-trivial and is often not enforced in polynomial interpolation methods.

\section{The Iterated Piecewise Quartic Polynomial Interpolation (IPQPI) Algorithm}

Our goal is to maximize the smoothness of the forward curve by minimizing its average curvature ${ }^{6}$

$$
\min _{f} \int\left(f^{\prime \prime}(t)\right)^{2} d t
$$

We model the forward curve as a polynomial spline

$$
f(t)=\sum_{k=0}^{K}=x_{i, k} t^{k}, \text { when } t \in\left(T_{i-1}, T_{i}\right]
$$

\footnotetext{
${ }^{6}$ In our experience, this method produces superior results to the approach of [31] who minimizes $\int\left(f^{\prime}(t)\right)^{2} d t$ which is the average slope of the forward curve or the curvature of the spot curve.
} 

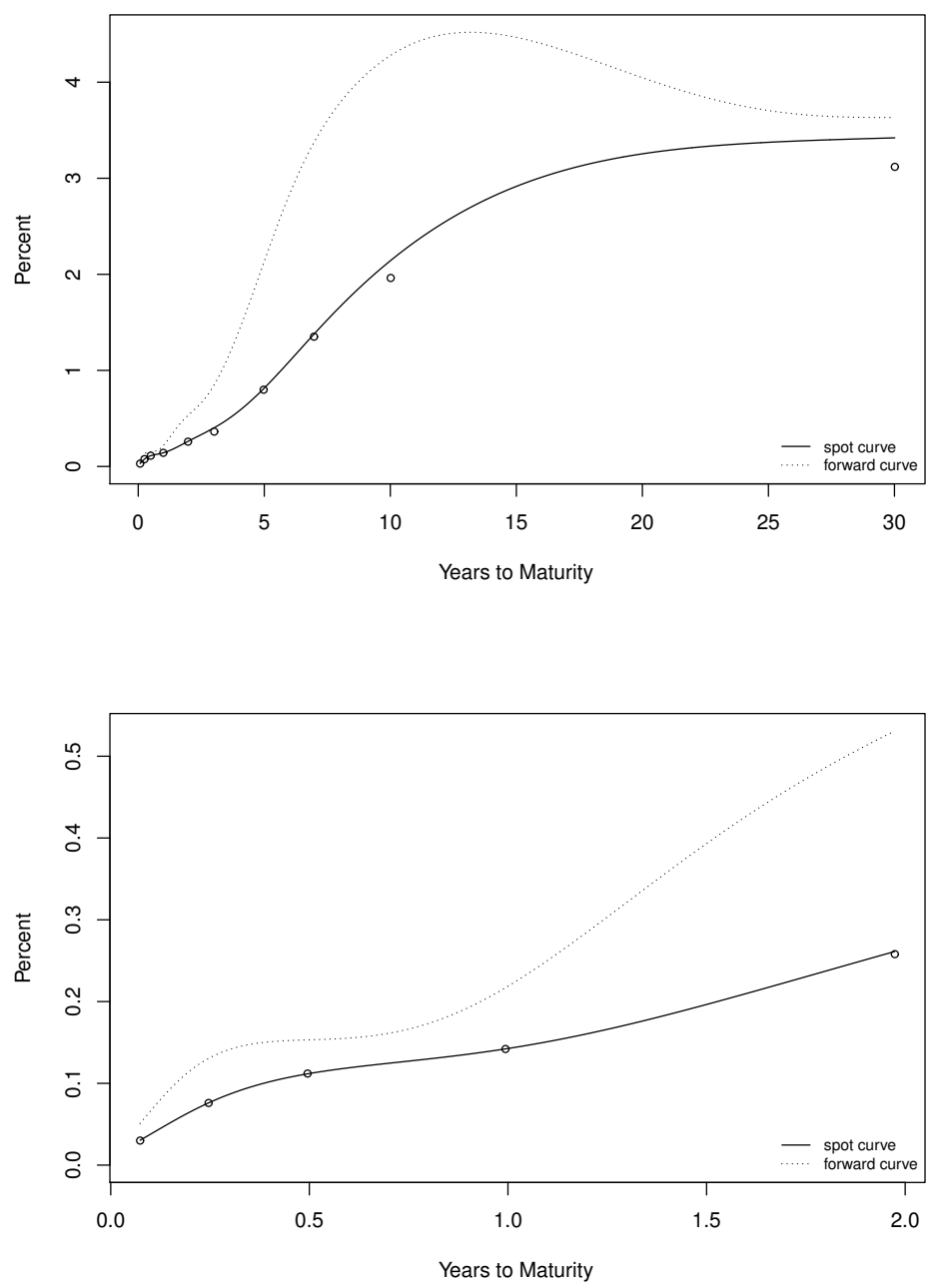

Figure 1. Computed spot (solid line) and forward rate (dotted line) curves for the yield curve data of February 10, 2012 shown in Table 1. Circles indicate the observed ask yields using discrete compounding. The top figure shows the curves for all maturities and the bottom figure shows the curves for maturities up to two years.

where the knots $T_{i}$ are the maturities of the $m$ securities with $T_{0}=0$ and the $x_{i, k}$ are the $K+1$ coefficients for polynomial segment $i$ that we will denote as $f_{i}(t)$.

We constrain the polynomial spline so that:

1. all securities are priced accurately;

2. the boundary conditions $f(0)=f_{0}$ and (3) are satisfied;

3 . there is continuity of the spline function at the knots

$$
f_{i}\left(T_{i}\right)=f_{i+1}\left(T_{i}\right), i=1, \ldots, m ;
$$

4. there is first-order differentiability of the spline function at the knots

$$
f_{i}^{\prime}\left(T_{i}\right)=f_{i+1}^{\prime}\left(T_{i}\right), i=1, \ldots, m ;
$$

5. there is second-order differentiability of the spline function at the knots

$$
f_{i}^{\prime \prime}\left(T_{i}\right)=f_{i+1}^{\prime \prime}\left(T_{i}\right), i=1, \ldots, m .
$$


This is a variational calculus problem first presented by [1]. An error in the original proof is corrected by [2] who also prove the following proposition:

Proposition 1 [Lim \& Xiao] When $f(0)=f_{0}$ is known and $f^{\prime}\left(T_{m}\right)=0$, the function of the maximum smoothness forward curve is a fourth-order polynomial spline and is second-order continuously differentiable in the range $\left(0, T_{m}\right)$.

Thus, the maximally smooth forward curve is given by the quartic polynomial spline

$$
f_{i}(t)=x_{i, 4} t^{4}+x_{i, 3} t^{3}+x_{i, 2} t^{2}+x_{i, 1} t+x_{i, 0}
$$

when $T_{i-1} \leq t \leq T_{i}$ and $i=1 \ldots, m$.

The proof of the proposition is quite general and easily extended to the case of coupon bonds but the algorithm proposed by [2] is specific to the zero coupon bond case and must be extended to cover the case of coupon bearing bonds.

The price of a zero coupon bond with maturity $T_{i}$ and face value $\$ 1$ is simply the value of the discount rate at that maturity, $P_{i}\left(T_{i}\right)=\delta\left(T_{i}\right)$, which, from (2a), implies

$$
-\ln \left(P_{i}\left(T_{i}\right)\right)=y\left(T_{i}\right) T_{i}=\sum_{j=1}^{i} \int_{T_{j-1}}^{T_{j}} f_{j}(t) d t .
$$

Since zero coupon bonds have a single cash flow at the maturity date for which there is a market price and thus a known spot rate, the algorithm for finding a smooth forward curve reduces to an interpolation problem.

Because $f_{i}(t)$ is a quartic polynomial, the objective function (4) will be quadratic in the coefficients $x$. Furthermore, all of the constraints can be written as linear expressions of the coefficients so that the constrained optimization problem can be written in the form

$$
\min _{x, \lambda} Z(x, \lambda)=x^{\prime} H x+\lambda^{\prime}(A x-B)
$$

where $\lambda$ is the vector of Lagrange multipliers and $x$ is the $5(m+1)$ vector of unknown coefficients. The elements of matrices $H_{5(m+1) \times 5(m+1)}$ and $A_{(4 m+5) \times 5(m+1)}$ are all functions of the known maturities $T_{i}, i=0, \ldots, m$, and the vector $B_{(4 m+5) \times 1}$ is a function of the observed asset prices and boundary constraints. We impose the terminal condition by adding an $(m+1)^{t h}$ knot such that $f_{m+1}(t)=x_{m+1,0}$ for $t \geq T_{m}$. This and the initial boundary condition together imply the five restrictions: $x_{1,0}=f_{0}$ and $x_{m+1,4}=x_{m+1,3}=x_{m+1,2}=x_{m+1,1}=0 . f_{0}$ is estimated by backward linear interpolation using the $\left(T_{1}, y_{1}\right),\left(T_{2}, y_{2}\right)$ knots.

There are a total of $5(m+1)$ parameters with $4 m+5$ restrictions (the 5 boundary restrictions and the four sets of $m$ restrictions $1,3,4$ and 5 specified above) leaving $m$ degrees of freedom. In their original derivation, [1] incorrectly imposes two additional restrictions per polynomial so they have only $3(m+1)$ coefficients to estimate which are actually fewer than the number of restrictions. Consequently, they drop the twice-differentiability condition ( 5 above) and impose only 2 terminal polynomial restrictions so that their model is exactly identified. However, these incorrect restrictions on the polynomials produce forward curves that do not have the desired maximum smoothness property. These errors are corrected by [2] but in the process they also introduce an error in the terminal condition $f^{\prime}\left(T_{m}\right)=0$ of the forward curve. Details of our corrected version of the zero coupon algorithm are presented in the Appendix. Hereafter we will refer to this algorithm as the LX algorithm. The critical point is that the LX portion of our algorithm can be solved using simple linear algebra methods.

Extending the algorithm to account for coupon bonds in the list of securities considerably complicates the problem and makes the solution algorithm highly nonlinear [35]. A coupon bond has a cash flow of the face amount plus the coupon payment at the maturity date but the observed market price of the bond also includes the present discounted value of the semi-annual coupon payments between the settlement date and the maturity date. Since the spot rate for these intermediate coupon payments is not observed, the spot rate at the maturity dates of the coupon bonds is also unknown. ${ }^{7}$ To find the forward

\footnotetext{
${ }^{7}$ What is observed is the yield-to-maturity of the bond but this is not sufficient to compute the spot rates at the coupon and maturity dates of the bond.
} 
curve in the coupon bond case we must simultaneously estimate the spot rates for all maturity dates and interpolate between these dates in such a way that all securities are accurately priced. ${ }^{8}$

Our approach is to iteratively apply the linear LX algorithm described above while updating the estimated spot rates at the knots using a global optimization method. In the first step we use the observed yield-to-maturity of each coupon bond to approximate the spot rates at the knots and apply the LX algorithm. This produces estimates of the coefficients $x$ that correctly price the zero coupon bonds up to one year in maturity but generate large pricing errors in the coupon bonds of greater than one year maturity.

The estimated price of the $i^{\text {th }}$ coupon bond with face value $\$ 100$ is

$$
\widehat{P}_{i}=\sum_{j=1}^{n_{i}-1} \widehat{\delta}\left(t_{i}(j)\right) \frac{C_{i}}{2}+\widehat{\delta}\left(T_{i}\right)\left(\frac{C_{i}}{2}+100\right)
$$

where $n_{i}$ is the number of remaining coupon payments at half the coupon rate for bond $i$ which pay at dates $t_{i}(j) . \widehat{\delta}\left(t_{i}(j)\right)$ is the estimated discount rate at date $t_{i}(j)$ as estimated using the coefficients from the initial LX step.

Next we update the spot rates for the coupon bond knots using

$$
\min _{\widehat{y}_{i}} \sum_{i}\left(P_{i}-\widehat{P}_{i}\right)^{2}
$$

where $\widehat{y}_{i}$, defined by $\widehat{\delta}\left(T_{i}\right)=\exp \left(-\widehat{y}_{i} T_{i}\right)$, are the updated spot rates at the knots and which can be computed as

$$
\widehat{y}_{i}=-\frac{1}{T_{i}} \ln \left(\frac{P_{i}-\sum_{j=1}^{n_{i}-1} \widehat{\delta}\left(t_{i}(j)\right) \frac{C_{i}}{2}}{\frac{C_{i}}{2}+100}\right) .
$$

Using these updated spot rates, we re-run the LX algorithm and update the spot rates using (10) and iterate until all spot rates converge and the prices of all securities are computed to arbitrary precision. The problem is a well-posed, quadratic, concave program so convergence to a unique minimum is assured.

Pseudo code for the IPQPI algorithm is shown in Figure 2. Importantly, although the algorithm goes through many iterations to compute the forward curve, each iteration involves the solution of a linear system of equations using the LX algorithm so the overall algorithm is very fast. Even when coded in an interpreted language such as R, the algorithm executes on a standard PC in less than one second. If coded in a lower level language such as FORTRAN or $\mathrm{C}++$ the algorithm will execute in fractions of a second.

The yield curve data in Table 1 that produces the spot curve shown in Figure 1 has a fairly "normal" shape but, even in this case, the implied forward curve is not unimodal. This shape is impossible for simpler models such as the Nelson-Siegel model [15] to capture and even the more flexible Svensson model [17] has difficulty adequately modeling the curve. Both of these methods will produce sizable pricing errors for the coupon bonds.

\section{An Application to an Inverted Forward Curve}

During periods of economic transitions the shape of the yield curve can become very complex. Figure 3 shows our computed yield curve from August 31, 1981, just as the U.S. economy was entering a severe recession and interest rates were very high. The spot curve is inverted ${ }^{9}$ and the forward curve is

\footnotetext{
8 The commonly used method of linear bootstrapping does produce zero pricing errors for the included securities but also produces a piece-wise linear spot curve and thus a discontinuous forward curve. This introduces false arbitrage signals into the pricing of fixed-income derivatives and so is an unacceptable method for most applications.

${ }^{9}$ When a recession is looming investors attempt to sell their short bonds and buy long bonds to lock in the rates before the declining economy drags down rates. This process drives down the prices of short bonds, and thus drives their yields up, while driving up the price and down the yields of long bonds. When the yield on long bonds is lower than the yield on short bonds we say that the yield curve is "inverted". This typically occurs several months before the real sectors of the economy begin to decline.
} 


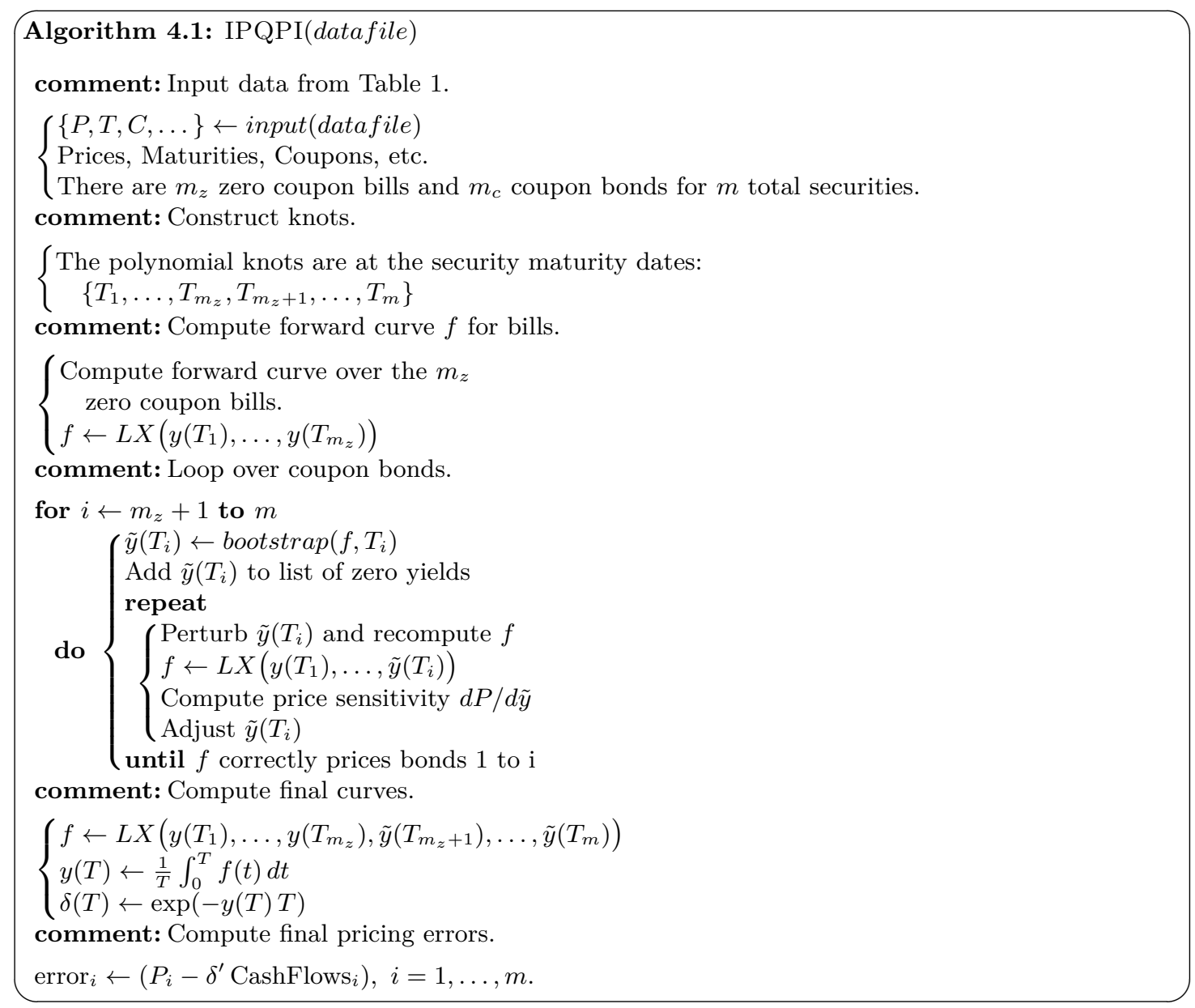

Figure 2. Pseudocode for the Iterated Piecewise Quartic Polynomial Interpolation (IPQPI) algorithm

multimodal with a very complex shape for maturities less than five years. This was also a period of very high inflation and, consequently, high bond yields.

Inverted yield curves can be difficult to model because the fit can be very sensitive to the terminal conditions, particularly the condition on long bonds. Notice from Figure 3 that the forward curve for maturities up to five years is very complicated and multimodal. Note also that our estimated forward curve levels out at the maturity of 30 years so that the projected spot curve will not become negative for out-of-sample maturities. This yield curve requires an order of magnitude more iterations to compute to the same accuracy as the yield curve in Figure 1 but still executes in under five seconds when coded in $\mathrm{R}$ and running on a personal computer.

Commonly used algorithms such as [15] or [17] do a very poor job of fitting yield curves with shapes as complicated as Figure 3 and can produce large pricing errors in the underlying securities. Recently, [44] extended the Nelson-Siegel model to an arbitrage-free framework and show improved performance. Note that our model does not produce negative forward rates and thus will also be arbitrage-free. More complex algorithms such as [35] can become unstable and require long execution times when applied to inverted yield curves. Finally, it is also worth noting that the lower graph of Figure 3 illustrates nicely how different spot rates can be when using continuous compounding (solid curve) and discrete bond equivalent yields (circles) when interest rates are very high. 

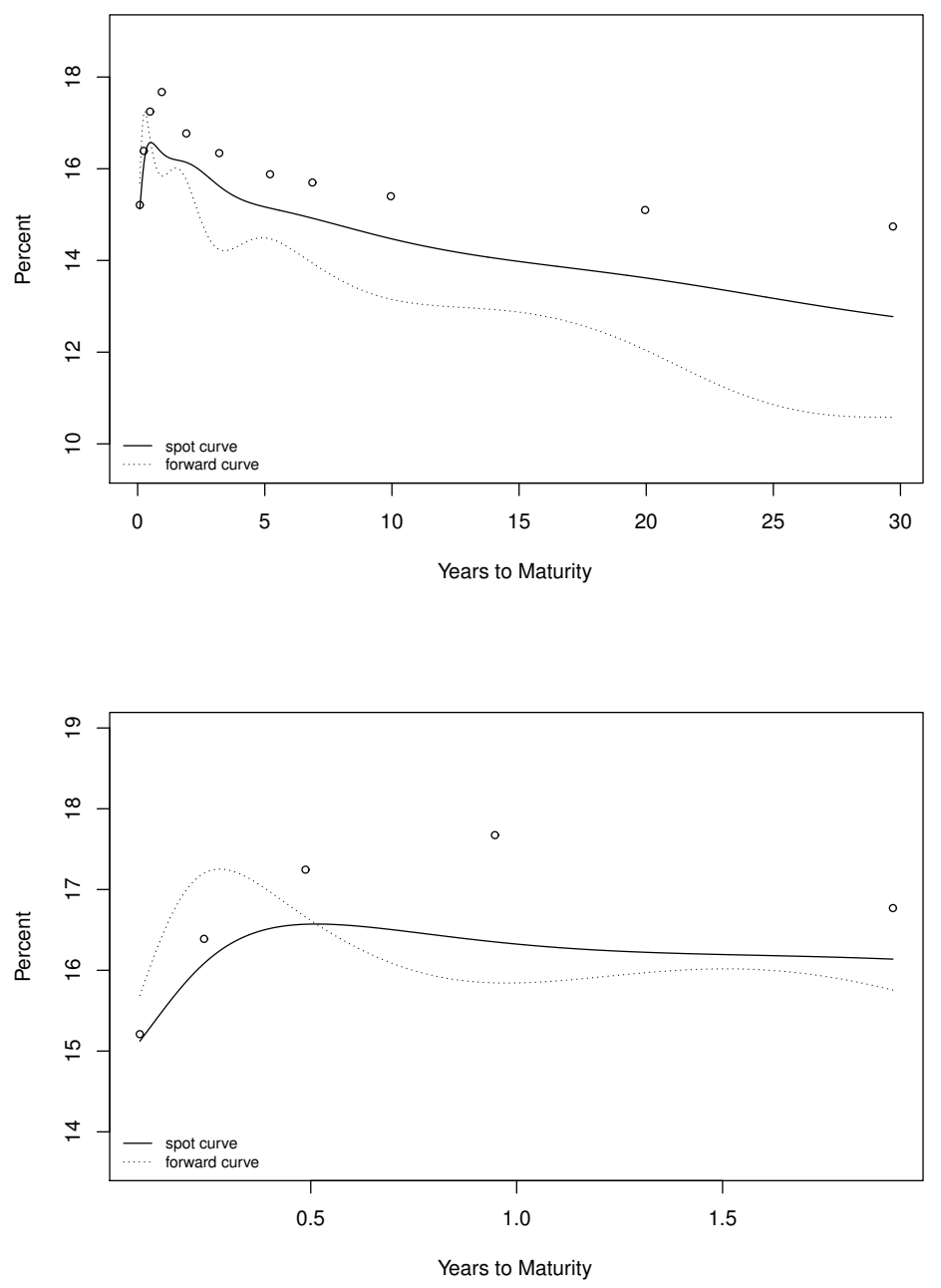

Figure 3. Computed spot (solid line) and forward rate (dotted line) curves for the inverted yield curve on August 31, 1981. Circles indicate the observed ask yields using discrete compounding. The top figure shows the curves for all maturities and the bottom figure shows the curves for maturities up to two years.

\section{Conclusions}

Over the course of time, fixed income analysts will experiment with many term structure models. New and exotic algorithms come and go on a fairly regular basis as attempts are made to achieve the five stated goals outlined in Section 2. The Iterated Piecewise Quartic Polynomial Interpolation algorithm does an excellent job of satisfying the goals of no-arbitrage, computational tractability, smooth forward curves, and accurate pricing but, admittedly, comprises on the goal of being able to be derived from first principles of current dynamic, stochastic, general equilibrium financial economic theory. As practitioners, we are reluctant but willing to make this sacrifice.

Our algorithm is fast, simple and highly accurate making it a practical tool for portfolio managers and traders. Computation speed is important to a trader who needs fast quotes based upon up-to-the-second yield curve data and to portfolio managers who can use the derived discount function to price a large segment of fixed income securities on-the-fly. A typical fixed income portfolio may consist of hundreds to thousands of securities and the portfolio value often must be computed within intervals of seconds. When using slower algorithms, it is not unusual for traders and portfolio managers to price different security 
classes within a portfolio using "approximate" models in order to make the computations fast enough for practical use. This creates discrepancies in asset prices across security classes that may not be resolved until books are closed in the late afternoon. One of the primary motivations for our algorithm was to be able to produce reliable and consistent discount functions within seconds.

The simplicity of our algorithm is also of practical importance. Traders, portfolio managers and clients, can understand polynomial interpolation quickly and the adjustment and iteration steps are easily explained. Complex affine and other nonlinear models must often be fit using nonlinear maximum likelihood methods that are computationally fragile and very difficult to debug and explain to clients who, after all, are the people providing the money. Our approach is to use an algorithm no more complex than necessary to solve the problem but no less complex than necessary to solve the problem accurately.

Pricing accuracy is paramount for traders and fund managers. We routinely price fixed income securities to a precision within several decimal points which ensures that multimillion dollar positions are accurate to within a penny. The algorithm is easily modified to handle more complex assets such as average-based forward contracts [40] or to include non-negativity constraints or local stability conditions to minimize hedging costs [4].

\section{References}

1. K. Adams and D. van Deventer, "Fitting yield curves and forward rate curves with maximum smoothness," The Journal of Fixed Income, vol. 4, no. 1, pp. 52-62, 1994.

2. K. G. Lim and Q. Xiao, "Computing maximum smoothness forward rate curves," Journal of Statistics and Computing, vol. 12(3), pp. 275-279, 2002.

3. P. Diament, "Semi-empirical smooth fit to the treasury yield curve," The Journal of Fixed Income, vol. 3, no. 1, pp. 55-70, 1993. [Online]. Available: http://www.iijournals.com/doi/abs/10.3905/jfi.1993.408073

4. P. S. Hagan and G. West, "Interpolation methods for curve construction," Applied Mathematical Finance, vol. 13 (2), pp. 89-129, 2006.

5. K. Adams, "Smooth interpolation of zero curves," ALGO Research Quarterly, vol. 4, no. 1/2, pp. 11-22, March/June 2001.

6. F. A. Lutz, "The structure of interest rates," The Quarterly Journal of Economics, vol. 55 (1), pp. 36-63, 1940.

7. J. M. Culbertson, "The term structure of interest rates," The Quarterly Journal of Economics, vol. 71, no. 4, pp. $485-517,1957$.

8. R. E. Lucas Jr., "Asset prices in an exchange economy." Econometrica, vol. 46, no. 6, pp. 1429-1445, November 1978.

9. J. C. Cox, J. E. Ingersoll Jr., and S. A. Ross, "A re-examination of traditional hypotheses about the term structure of interest rates," The Journal of Finance, vol. Vol. 36, No. 4, pp. 769-799, 1981.

10. J. C. Cox, J. Ingersoll Jr., Jonathan E., and S. A. Ross, "An intertemporal general equilibrium model of asset prices," Econometrica, vol. 53, no. 2, pp. 363-384, 1985.

11. O. A. Vasicek, "An equilibrium characterization of the term structure," Journal of Financial Economics, vol. 40, pp. 319-325, 1977.

12. M. J. Brennan and E. S. Schwartz, "A continuous-time approach to the pricing of bonds," Journal of Banking and Finance, vol. 3, pp. 135-155, 1979.

13. T. C. Langetieg, "A multivariate model of the term structure," The Journal of Finance, vol. 35, no. 1, pp. 71-97, 1980.

14. T. S. Y. Ho and S.-B. Lee, "Term structure movements and pricing interest rate contingent claims," The Journal of Finance, vol. 41, no. 5, pp. 1011-1029, 1986.

15. C. R. Nelson and A. F. Siegel, "Parsimonious modeling of yield curves," The Journal of Business, vol. 60 (4), pp. $473-489,1987$.

16. F. A. Longstaff, "Time varying term premia and traditional hypotheses about the term structure," The Journal of Finance, vol. 45, no. 4, pp. 1307-1314, 1990.

17. L. E. O. Svensson, "Estimating forward interest rates with the extended nelson \& siegel method," Sveriges Riksbank Quarterly Review, vol. 3, pp. 13-26, 1995.

18. F. X. Diebold and C. Li, "Forecasting the term structure of government bond yields," Journal of Econometrics, vol. 130, pp. 337-364, 2006.

19. D. Heath, R. Jarrow, and A. Morton, "Bond pricing and the term structure of interest rates: A new methodology for contingent claims valuation," Econometrica, vol. 60 (1), pp. 77-107, 1992. 
20. A. Brace and M. Musiela, "A multifactor gauss markov imiplementatin of heath, jarrow, and morton," Mathematical Finance, vol. 4, no. 3, pp. 259-283, 1994.

21. Q. Dai and K. J. Singleton, "Specification analysis of affine term structure models," The Journal of Finance, vol. 55(5), pp. 1943-1978, 2000.

22. F. Audrino and E. De Giorgi, "Beta regimes for the yield curve," Journal of Financial Econometrics, vol. 5, no. 3, pp. 456-490, 2007.

23. J. H. Christensen, F. X. Diebold, and G. D. Rudebusch, "An arbitrage-free generalized nelson-siegel term structure model," The Econometrics Journal, vol. 12, no. 3, pp. C33-C64, 2009.

24. M. P. Laurini and L. K. Hotta, "Bayesian extensions to diebold-li term structure model," International Review of Financial Analysis, vol. 19, no. 5, pp. 342-350, 2010.

25. J. H. Christensen, F. X. Diebold, and G. D. Rudebusch, "The affine arbitrage-free class of nelson-siegel term structure models," Journal of Econometrics, vol. 164, no. 1, pp. 4-20, 2011.

26. C. Hevia, M. Gonzalez-Rozada, M. Sola, and F. Spagnolo, "Estimating and forecasting the yield curve using a markov switching dynamic nelson and siegel model," Journal of Applied Econometrics, vol. 30, no. 6, pp. 987-1009, 2015.

27. J. H. McCulloch, "The tax-adjusted yield curve," The Journal of Finance, vol. 30 (3), pp. 811-830, 1975.

28. O. A. Vasicek and H. G. Fong, "Term structure modeling using exponential splines," The Journal of Finance, vol. 37 (2), pp. 339-348, 1982.

29. G. S. Shea, "Interest rate term structure estimation with exponential splines: A note," The Journal of Finance, vol. 40 (1), pp. 319-325, 1985.

30. T. S. Coleman, L. Fisher, and R. G. Ibbotson, "Estimating the term structure of interest rates from data that include the prices of coupon bonds," The Journal of Fixed Income, vol. 2, no. 2, pp. 85-116, 1992. [Online]. Available: http://www.iijournals.com/doi/abs/10.3905/jfi.1992.408048

31. V. Frishling and J. Yamamura, "Fitting a smooth forward rate curve to coupon instruments," The Journal of Fixed Income, vol. 6, no. 2, pp. 97-103, 1996. [Online]. Available: http: //www.iijournals.com/doi/abs/10.3905/jfi.1996.408174

32. S. A. Mansi and J. H. Phillips, "Modeling the term structure from the on-the-run treasury yield curve," The Journal of Financial Research, vol. 24 (4), pp. 545-564, 2001.

33. J. V. Jordan and S. A. Mansi, "Term structure estimation from on-the-run treasuries," Journal of Banking $\mathcal{E}$ Finance, vol. 27, 2003.

34. M. Fisher, "Modeling the term structure of interest rates: An introduction," Federal Reserve Bank of Atlanta Economic Review, vol. 89, no. 3, pp. 41-62, 2004.

35. J. Manzano and J. Blomvall, "Positive forward rates in the maximum smoothness framework," Quantitative Finance, vol. 4, no. 2, pp. 221-232, APR 2004.

36. R. Gimeno and J. M. Nave, "Genetic algorithm estimation of interest rate term structure," Banco de España, Tech. Rep. 0634, Dec. 2006.

37. F. Fernández-Rodríguez, "Interest rate term structure modeling using free-knot splines," The Journal of Business, vol. 79, no. 6, pp. 3083-3099, 2006.

38. B. Jungbacker, S. J. Koopman, and M. Wel, "Smooth dynamic factor analysis with application to the us term structure of interest rates," Journal of Applied Econometrics, vol. 29, no. 1, pp. 65-90, 2014.

39. B. G. Turan and A. k. Karagozoglu, "Pricing eurodollar futures options using the edt term structure model: The effects of yield curve smoothing," The Journal of Futures Markets, vol. 20 (3), pp. 293-306, 2000.

40. F. E. Benth, S. Koekkebakker, and F. Ollmar, "Extracting and applying smooth forward curves from average-based commodity contracts with seasonal variation," Journal of Derivatives, vol. 15, no. 1, pp. 52-66, 2007.

41. M. Ioannides, "A comparison of yield curve estimation techniques using UK data," Journal of Banking $E^{3}$ Finance, vol. 27, no. 1, pp. 1-26, JAN 2003.

42. P. Veronesi, Fixed Income Securities: Valuation, Risk, and Risk Management. Hoboken, New Jersey: Wiley, 2010.

43. P. Wilmott, Derivatives: The theory and practice of financial engineering. New York, New York: Wiley, 1998.

44. R. Chen and K. Du, "A generalised arbitrage-free Nelson-Siegel model: The impact of unspanned stochastic volatility," Finance Research Letters, vol. 10, no. 1, pp. 41-48, March 2013.

\section{Appendix: Interpolating the Zero Coupon Curve-The LX Algorithm}

The procedure for interpolating the zero coupon portion of the yield curve is similar to that described in [2] except that we handle the terminal condition on the forward curve differently. Our approach adds one additional segment to the piecewise spline function with some additional coefficient restrictions on the 
terminal spline. Since the matrix sizes differ to reflect these changes, we provide the details of this step here.

In the first subsection of the appendix we show how to construct the objective function as a quadratic expression. In the second subsection we construct the constraints as linear equations and in the final subsection we show how to solve the system.

\section{A.1 Specifying the Objective Function}

Noting that the objective function is piecewise with knots at the maturity of each security. Let $f_{i}(t)=$ $a_{i} t^{4}+b_{i} t^{3}+c_{i} t^{2}+d_{i} t+e_{i}$ denote the quartic polynomial between knot $T_{i-1}$ to knot $T_{i}$ so that $f_{i}^{\prime \prime}(t)=$ $12 a_{i} t^{3}+6 b_{i} t^{2}+2 c_{i}$. Then the objective function (4) may be written as

$$
\begin{aligned}
\int_{0}^{T_{m+1}}\left(f^{\prime \prime}(t)\right)^{2} d t \\
=\sum_{i=1}^{m+1} \int_{T_{i-1}}^{T_{i}} f_{i}^{\prime \prime}(t) d t \\
=\sum_{i=1}^{m+1} \int_{T_{i-1}}^{T_{i}}\left(12 a_{i} t^{2}+6 b_{i} t+2 c_{i}\right)^{2} d t \\
=\sum_{i=1}^{m+1}\left(\frac{144}{5} \Delta_{i}^{5} a_{i}^{2}+36 \Delta_{i}^{4} a_{i} b_{i}+12 \Delta_{i}^{3} b_{i}^{2}\right. \\
\left.+16 \Delta_{i}^{3} a_{i} c_{i}+12 \Delta_{i}^{2} b_{i} c_{i}+4 \Delta_{i} c_{i}^{2}\right) \\
=\sum_{i=1}^{m+1} x_{i}^{\prime} h_{i} x_{i} \\
=\boldsymbol{X}^{\prime} \boldsymbol{H} \boldsymbol{X}
\end{aligned}
$$

where $\Delta_{i}^{n}=T_{i}^{n}-T_{i-1}^{n}$,

$$
\begin{aligned}
& x_{i}=\left(\begin{array}{c}
a_{i} \\
b_{i} \\
c_{i} \\
d_{i} \\
e_{i}
\end{array}\right), h_{i}=\left(\begin{array}{ccccc}
\frac{144}{5} \Delta_{i}^{5} & 18 \Delta_{i}^{4} & 8 \Delta_{i}^{3} & 0 & 0 \\
18 \Delta_{i}^{4} & 12 \Delta_{i}^{3} & 6 \Delta_{i}^{2} & 0 & 0 \\
8 \Delta_{i}^{3} & 6 \Delta_{i}^{2} & 4 \Delta_{i} & 0 & 0 \\
0 & 0 & 0 & 0 & 0 \\
0 & 0 & 0 & 0 & 0
\end{array}\right) \\
& \boldsymbol{X}=\left(\begin{array}{c}
x_{1} \\
\vdots \\
x_{m+1}
\end{array}\right)_{5(m+1) \times 1}, \\
& \boldsymbol{H}=\left(\begin{array}{ccccc}
h_{1} & 0 & 0 & \cdots & 0 \\
0 & h_{2} & 0 & \cdots & 0 \\
\vdots & & \ddots & \vdots \\
0 & 0 & 0 & \cdots & h_{m+1}
\end{array}\right)_{5(m+1) \times 5(m+1)},
\end{aligned}
$$

and each of the 0's as well as $h_{m+1}$ is a $5 \times 5$ matrix of zeros.

Recall that we have $m$ securities with maturities $T_{1}, \ldots, T_{m}$ where $m$ is the number of securities. We do not consider the settlement date in this list so $T_{1}$ is the maturity of the first real security - the one-week LIBOR in our example. We define $T_{0}=0$. Note that we have added an $m+1$ knot in (A.1). The reason for this additional knot is to impose the terminal condition on the forward curve which is incorrectly handled in [2]. This turns out to be more complicated than expected and we will discuss this correction in more detail below.

The important observation to make is that the objective function is quadratic so that the gradient is linear in the coefficients. We will now show that the constraint functions are also linear functions of the coefficients. 


\section{A.2 Specifying the Constraint Functions}

The zero-coupon bond price given by (2a) may be written as

$$
\begin{aligned}
-\ln & \delta\left(T_{j}\right)=\int_{0}^{T_{j}} f(t) d t \\
= & \sum_{i=1}^{j} \int_{T_{i-1}}^{T_{i}} f_{i}(t) d t \\
= & \sum_{i=1}^{j} \int_{T_{i-1}}^{T_{i}}\left(a_{i} t^{4}+b_{i} t^{3}+c_{i} t^{2}+d_{i} t+e_{i}\right) d t \\
= & \sum_{i=1}^{j}\left(\frac{1}{5} a_{i} T_{i}^{5}+\frac{1}{4} b_{i} T_{i}^{4}+\frac{1}{3} c_{i} T_{i}^{3}+\frac{1}{2} d_{i} T_{i}^{2}+e_{i} T_{i}\right)
\end{aligned}
$$

so that the $\log$ of the zero-coupon bond price is linear in the coefficients $x$.

Recalling our previous notation $\Delta_{j}^{n}=T_{j}^{n}-T_{j-1}^{n}$, we can write the difference of the log prices of two consecutive zero coupon bonds as

$$
-\ln \left(\frac{\delta\left(T_{j}\right)}{\delta\left(T_{j-1}\right)}\right)=\frac{1}{5} \Delta_{j}^{5} a_{j}+\frac{1}{4} \Delta_{j}^{4} b_{j}+\frac{1}{3} \Delta_{j}^{3} c_{j}+\frac{1}{2} \Delta_{j}^{2} d_{j}+\Delta_{j} e_{j} .
$$

The full set of pricing constraints for all $m$ bonds may then be written in matrix form as

$$
\boldsymbol{A}_{1} \boldsymbol{X}=\boldsymbol{B}_{1}
$$

where

$$
\begin{aligned}
\boldsymbol{A}_{1} & =\left(\begin{array}{cccc}
D T_{1} & 0_{1 \times 5} & \cdots & 0_{1 \times 5} \\
0_{1 \times 5} & D T_{2} & \cdots & 0_{1 \times 5} \\
\vdots & \vdots & \ddots & \vdots \\
0_{1 \times 5} & 0_{1 \times 5} & \cdots & D T_{m}
\end{array}\right)_{m \times 5(m+1)}, \\
D T_{j} & =\left(\frac{1}{5} \Delta_{j}^{5}, \frac{1}{4} \Delta_{j}^{4}, \frac{1}{3} \Delta_{j}^{3}, \frac{1}{2} \Delta_{j}^{2}, \Delta_{j}\right)_{1 \times 5},
\end{aligned}
$$

and

$$
\boldsymbol{B}_{1}=\left(\begin{array}{c}
\ln \left(\delta\left(T_{1}\right) / \delta\left(T_{0}\right)\right) \\
\ln \left(\delta\left(T_{2}\right) / \delta\left(T_{1}\right)\right) \\
\vdots \\
\ln \left(\delta\left(T_{m}\right) / \delta\left(T_{m-1}\right)\right)
\end{array}\right)_{m \times 1}
$$

Next we impose constraints to ensure that the forward curve remains smooth as it transits through knot points in the piecewise polynomial approximation. To ensure continuity at the knot points we require that the forward rate at $\operatorname{knot} T_{i}$ has the same value at that either computed using the left-side polynomial or the right-side polynomial. Thus, we impose

$$
f_{i+1}\left(T_{i}\right)=f_{i}\left(T_{i}\right), i=1, \ldots, m
$$

or

$$
\left(a_{i+1}-a_{i}\right) T_{i}^{4}+\left(b_{i+1}-b_{i}\right) T_{i}^{3}+\left(c_{i+1}-c_{i}\right) T_{i}^{2}+\left(d_{i+1}-d_{i}\right) T_{i}+\left(e_{i+1}-e_{i}\right)=0, i=1, \ldots, m .
$$

Define $T 4_{i}=\left(T_{i}^{4}, T_{i}^{3}, T_{i}^{2}, T_{i}, 1\right)_{1 \times 5}$ and write all $m$ of these constraints in matrix form as

$$
\boldsymbol{A}_{2} X=\boldsymbol{B}_{2}
$$


where

$$
\boldsymbol{A}_{2}=\left(\begin{array}{ccccccc}
-T 4_{1} & T 4_{1} & 0_{1 \times 5} & 0_{1 \times 5} & \cdots & 0_{1 \times 5} & 0_{1 \times 5} \\
0_{1 \times 5} & -T 4_{2} & T 4_{2} & 0_{1 \times 5} & \cdots & 0_{1 \times 5} & 0_{1 \times 5} \\
\vdots & \vdots & \vdots & \vdots & \ddots & \vdots & \vdots \\
0_{1 \times 5} & 0_{1 \times 5} & 0_{1 \times 5} & 0_{1 \times 5} & \cdots & -T 4_{m} & T 4_{m}
\end{array}\right)
$$

is an $m \times 5(m+1)$ matrix and $\boldsymbol{B}_{2}$ is an $m \times 1$ vector of zeros.

To impose differentiability at the knots we require

$$
f_{i+1}^{\prime}\left(T_{i}\right)=f_{i}^{\prime}\left(T_{i}\right), i=1, \ldots, m
$$

or

$$
4\left(a_{i+1}-a_{i}\right) T_{i}^{3}+3\left(b_{i+1}-b_{i}\right) T_{i}^{2}+2\left(c_{i+1}-c_{i}\right) T_{i}+\left(d_{i+1}-d_{i}\right)=0, i=1, \ldots, m .
$$

Define $T 3_{i}=\left(4 T_{i}^{3}, 3 T_{i}^{2}, 2 T_{i}, 1,0\right)_{1 \times 5}$ and write all $m$ of these constraints in matrix form as

$$
\boldsymbol{A}_{3} \boldsymbol{X}=\boldsymbol{B}_{3} 0_{m \times 1}
$$

where

$$
\boldsymbol{A}_{3}=\left(\begin{array}{ccccccc}
-T 3_{1} & T 3_{1} & 0_{1 \times 5} & 0_{1 \times 5} & \cdots & 0_{1 \times 5} & 0_{1 \times 5} \\
0_{1 \times 5} & -T 3_{2} & T 3_{2} & 0_{1 \times 5} & \cdots & 0_{1 \times 5} & 0_{1 \times 5} \\
\vdots & \vdots & \vdots & \vdots & \ddots & \vdots & \vdots \\
0_{1 \times 5} & 0_{1 \times 5} & 0_{1 \times 5} & 0_{1 \times 5} & \cdots & -T 3_{m} & T 3_{m}
\end{array}\right)
$$

is an $m \times 5(m+1)$ matrix and $\boldsymbol{B}_{3}$ is an $m \times 1$ vector of zeros.

To ensure that the first derivatives of the forward curve are smooth at the knots we impose

$$
f_{i+1}^{\prime \prime}\left(T_{i}\right)=f_{i}^{\prime \prime}\left(T_{i}\right), i=1, \ldots, m,
$$

or

$$
12\left(a_{i+1}-a_{i}\right) T_{i}^{2}+6\left(b_{i+1}-b_{i}\right) T_{i}+2\left(c_{i+1}-c_{i}\right)=0, i=1, \ldots, m .
$$

Define $T 2_{i}=\left(12 T_{i}^{2}, 6 T_{i}, 2,1,0,0\right)_{1 \times 5}$ and write all $m$ of these constraints in matrix form as

$$
\boldsymbol{A}_{4} \boldsymbol{X}=\boldsymbol{B}_{4}
$$

where

$$
\boldsymbol{A}_{4}=\left(\begin{array}{ccccccc}
-T 2_{1} & T 2_{1} & 0_{1 \times 5} & 0_{1 \times 5} & \cdots & 0_{1 \times 5} & 0_{1 \times 5} \\
0_{1 \times 5} & -T 2_{2} & T 2_{2} & 0_{1 \times 5} & \cdots & 0_{1 \times 5} & 0_{1 \times 5} \\
\vdots & \vdots & \vdots & \vdots & \ddots & \vdots & \vdots \\
0_{1 \times 5} & 0_{1 \times 5} & 0_{1 \times 5} & 0_{1 \times 5} & \cdots & -T 2_{m} & T 2_{m}
\end{array}\right)
$$

is an $m \times 5(m+1)$ matrix and $\boldsymbol{B}_{4}$ is an $m \times 1$ vector of zeros.

To ensure the boundary condition $f(0)=y_{0}$, we simply impose $e_{1}=y_{0}$. The terminal boundary condition $f^{\prime}\left(T_{m}\right)=0$ is more difficult to impose. [2] uses the condition $d_{1}=0$ which is clearly incorrect. We impose the terminal condition by adding an additional $(m+1)^{s t}$ segment to the piecewise polynomial with the coefficient restrictions $a_{m+1}=b_{m+1}=c_{m+1}=d_{m+1}=0$ so that $f(t)=e_{m+1}$ for all $t>T_{m}$. The terminal height of the forward function is left unconstrained and the continuity and smoothness constraints described above will ensure a smooth transition to the zero slope of the forward curve at knot $T_{m}$.

These five boundary conditions may be written in matrix notation as

$$
\boldsymbol{A}_{5} \boldsymbol{X}=\boldsymbol{B}_{5}
$$

where

$$
\boldsymbol{A}_{5}=\left(\begin{array}{llllllllllll}
0 & 0 & 0 & 0 & 1 & 0 & \cdots & 0 & 0 & 0 & 0 & 0 \\
0 & 0 & 0 & 0 & 0 & 0 & \cdots & 1 & 0 & 0 & 0 & 0 \\
0 & 0 & 0 & 0 & 0 & 0 & \cdots & 0 & 1 & 0 & 0 & 0 \\
0 & 0 & 0 & 0 & 0 & 0 & \cdots & 0 & 0 & 1 & 0 & 0 \\
0 & 0 & 0 & 0 & 0 & 0 & \cdots & 0 & 0 & 0 & 1 & 0
\end{array}\right)_{5 \times 5(m+1)}
$$


and

$$
\boldsymbol{B}_{5}=\left(\begin{array}{c}
y_{0} \\
0 \\
0 \\
0 \\
0
\end{array}\right)
$$

Stacking all of these linear constraints gives

$$
\boldsymbol{A} \boldsymbol{X}=\boldsymbol{B}
$$

where

$$
\boldsymbol{A}=\left(\begin{array}{l}
\boldsymbol{A}_{1} \\
\boldsymbol{A}_{2} \\
\boldsymbol{A}_{3} \\
\boldsymbol{A}_{4} \\
\boldsymbol{A}_{5}
\end{array}\right)_{(4 m+5) \times 5(m+1)} \text { and }\left(\begin{array}{l}
\boldsymbol{B}_{1} \\
\boldsymbol{B}_{2} \\
\boldsymbol{B}_{3} \\
\boldsymbol{B}_{4} \\
\boldsymbol{B}_{5}
\end{array}\right)_{(4 m+5) \times 1}
$$

\section{A.3 Solving the System}

The constrained optimization problem may now be written in matrix notation as

$$
\min _{\boldsymbol{X}, \boldsymbol{\lambda}} Z(\boldsymbol{X}, \boldsymbol{\lambda})=\boldsymbol{X}^{\prime} \boldsymbol{H} \boldsymbol{X}+\boldsymbol{\lambda}^{\prime}(\boldsymbol{A} \boldsymbol{X}-\boldsymbol{B})
$$

where $\boldsymbol{\lambda}$ is the $4 m+5$ vector of Lagrange multipliers corresponding to the constraints.

The first-order conditions are

$$
\frac{\partial}{\partial \boldsymbol{X}} Z(\boldsymbol{X}, \boldsymbol{\lambda})=2 \boldsymbol{H} \boldsymbol{X}+\boldsymbol{A}^{\prime} \boldsymbol{\lambda}=0
$$

and

$$
\frac{\partial}{\partial \boldsymbol{\lambda}} Z(\boldsymbol{X}, \boldsymbol{\lambda})=\boldsymbol{A} \boldsymbol{H} \boldsymbol{X}-\boldsymbol{B}=0
$$

or

$$
\left(\begin{array}{cc}
2 \boldsymbol{H} & \boldsymbol{A}^{\prime} \\
\boldsymbol{A} & 0
\end{array}\right)\left(\begin{array}{c}
\boldsymbol{X} \\
\boldsymbol{\lambda}
\end{array}\right)=\left(\begin{array}{c}
0 \\
\boldsymbol{B}
\end{array}\right)
$$

from which we find the explicit solution

$$
\left(\begin{array}{c}
\boldsymbol{X}^{*} \\
\boldsymbol{\lambda}^{*}
\end{array}\right)=\left(\begin{array}{cc}
2 \boldsymbol{H} & \boldsymbol{A}^{\prime} \\
\boldsymbol{A} & 0
\end{array}\right)^{-1}\left(\begin{array}{l}
0 \\
\boldsymbol{B}
\end{array}\right)
$$

